\title{
Time Splitting and Grid Refinement Methods in the Lattice Boltzmann Framework for Solving a Reaction-Diffusion Process
}

\author{
Davide Alemani ${ }^{1}$, Bastien Chopard ${ }^{2}$, Josep Galceran ${ }^{3}$, and Jacques Buffle ${ }^{1}$ \\ ${ }^{1}$ CABE - Analytical and Biophysical Environmental Chemistry \\ Sciences II, 30 quai E. Ansermet, CH-1211 Geneva 4, Switzerland \\ \{Davide.Alemani, Jacques.Buffle\}@cabe.unige.ch \\ ${ }^{2}$ CUI - Computer Science Department \\ 24 rue General-Dufour CH-1211 Geneva 4, Switzerland \\ Bastien.Chopard@cui.unige.ch \\ ${ }^{3}$ ETSEA - Departament de Quimica, UdL \\ Av. Rovira Roure 191, 25198 Lleida, Spain \\ galceran@quimica.udl.es
}

\begin{abstract}
The paper shows how to combine together the Lattice Boltzmann Methods with the time splitting and the grid refinement techniques, in order to solve reaction-diffusion processes including very fast reaction dynamics, i.e. with time and length scales that vary in a wide range of values. The method is applied to the reaction prototype problem: $\mathrm{M}^{0} \leftarrow \mathrm{M}+\mathrm{L} \rightleftharpoons \mathrm{ML}$ with semi-infinite diffusion conditions and in presence of an electrode where Nernst + flux balance conditions are considered. Two important geometries are considered, planar and spherical, and off-lattice boundary conditions are set up, for general irregular and curved boundaries. We discuss the need, for some cases, of applying the time splitting and the grid refinement approach to have a numerical scheme more easily handled and to substantially reduce the computational time. Furthermore, we point out the physico-chemical conditions to apply the time splitting and the grid refinement to optimise accuracy and performance. In particular, we stress: a) the range of values of the relaxation BGK parameter to have the best performance in solving the pure diffusive scheme and $b$ ) the best values of the grid refinement factor to preserve a good accuracy and drastically reduce the time of computation and the memory usage.
\end{abstract}

\section{Introduction}

Environmental physicochemical problems usually involve a large number of processes that take place over a wide range of space and time scales. We will consider, in this paper, the typical reaction-diffusion process as:

$$
\mathrm{M}^{0} \leftarrow \mathrm{M}+\mathrm{L} \rightleftharpoons \mathrm{ML}
$$


wherein a metal ion $\mathrm{M}$ can react in solution with a ligand $\mathrm{L}$ forming a complex ML with given association and dissociation rate constants, $k_{\mathrm{a}}$ and $k_{\mathrm{d}}$ respectively. Furthermore, the metal ion is reduced at an electrode surface into $\mathrm{M}^{0}$. All the species diffuse with diffusion coefficients, $D_{\mathrm{M}}, D_{\mathrm{L}}, D_{\mathrm{ML}}$ and $D_{\mathrm{M}^{0}}$ respectively. Process (1) is a typical electrochemical problem and in environmental systems is a typical multi-scale problem. In fact, the reaction rate constants and the diffusion coefficients of the species can vary over a large range of values. Typically, the reaction constants $k_{\mathrm{a}}$ can take values between $10^{-6} \mathrm{~m}^{3} \mathrm{~mol}^{-1} \mathrm{~s}^{-1}$ and $10^{8} \mathrm{~m}^{3} \mathrm{~mol}^{-1} \mathrm{~s}^{-1}$, ranging from a complete inert to a fully dynamic case. On the other hand, the diffusion coefficients usually take values from $10^{-12} \mathrm{~m}^{2} \mathrm{~s}^{-1}$ to $10^{-9} \mathrm{~m}^{2} \mathrm{~s}^{-1}$. As a consequence, the reaction and the diffusion may occur at a different time and space scales. We shall focus the attention to the pure reactiondiffusion process, by applying a potential $E$ at the electrode surface. We take $E$ sufficiently negative relatively to the reduction-oxidation standard potential $E_{0}$. For instance, a value such that $E-E_{0}=-0.3 \mathrm{~V}$ is large enough in order to consider the electrode surface acting as a perfect sink for M.

The space-time scales of the system are described by four key parameters. The space scales of diffusion and reaction are described by the diffusion layer thickness $\delta$ and the reaction layer thickness $\mu$, respectively (see [1] for a complete explanation). In many cases, $\delta$, is expressed as [1]:

$$
\delta=\sqrt{\pi D_{\mathrm{M}} t}
$$

where $t$ is the total time in which diffusion occurs.

The thickness $\mu$ depends on the ratio of the diffusion rate of $\mathrm{M}$ over its recombination rate with $\mathrm{L}$ [1]:

$$
\mu=\sqrt{\frac{D_{\mathrm{M}}}{k_{\mathrm{a}} c_{\mathrm{L}}^{*}}}
$$

where $c_{\mathrm{L}}^{*}$ is the bulk concentration of $\mathrm{L}$.

The time scales of reaction and diffusion are described, respectively, by the recombination rate of $\mathrm{M}$ with $\mathrm{L}$ :

$$
\frac{1}{k_{\mathrm{a}} c_{\mathrm{L}}^{*}}
$$

and the diffusion rate of $\mathrm{M}$

$$
\frac{\delta^{2}}{D_{\mathrm{M}}}
$$

Relevant cases are those where the concentration of the complex ML is relatively large with respect to $\mathrm{M}$. In fact, let us consider the typical set of values wherein the bulk concentration of $\mathrm{L}, c_{\mathrm{L}}^{*}$ is in excess compared with the bulk concentration of $\mathrm{M}, c_{\mathrm{M}}^{*}: c_{\mathrm{M}}^{*}=10^{-3} \mathrm{~mol} \mathrm{~m}^{-3}, c_{\mathrm{L}}^{*}=1 \mathrm{~mol} \mathrm{~m}^{-3}, D_{\mathrm{M}}=10^{-9} \mathrm{~m}^{2} \mathrm{~s}^{-1}$, $k_{\mathrm{a}} c_{\mathrm{L}}^{*}=10^{8} \mathrm{~s}^{-1}$. By applying the potential $E$ at the electrode surface, the reaction is forced to be far from the chemical equilibrium and a diffusion gradient is established close to the electrode surface. After one second, the above mentioned parameters take the following values: $\mu=3.16 \cdot 10^{-9} \mathrm{~m}, \delta=5.6 \cdot 10^{-5} \mathrm{~m}$, 
$\left(k_{\mathrm{a}} c_{\mathrm{L}}^{*}\right)^{-1}=10^{-8} \mathrm{~S}$ and $\delta^{2} / D_{\mathrm{M}}=3.14 \mathrm{~s}$. Clearly, the reaction and the diffusion processes take place at very different scales. In order to numerically solve this kind of multi-scale process we use two numerical methods, the time splitting which allows us to discriminate fast and slow processes and the grid refinement which allows us to localise a fine grid only within regions wherein large variations of the concentration profiles are expected.

\subsection{Definition of the Problem}

The semi-infinite diffusion-reaction problem (11) is described by the following system of partial differential equations defined in an open set $\Omega(\forall t>0)$ :

$$
\begin{gathered}
\frac{\partial c_{\mathrm{M}}(x, t)}{\partial t}=D_{\mathrm{M}} \nabla^{2} c_{\mathrm{M}}(x, t)+R(x, t) \\
\frac{\partial c_{\mathrm{ML}}(x, t)}{\partial t}=D_{\mathrm{ML}} \nabla^{2} c_{\mathrm{ML}}(x, t)-R(x, t) \\
\frac{\partial c_{\mathrm{L}}(x, t)}{\partial t}=D_{\mathrm{L}} \nabla^{2} c_{\mathrm{L}}(x, t)+R(x, t)
\end{gathered}
$$

where

$$
R(x, t)=k_{\mathrm{d}} c_{\mathrm{ML}}(x, t)-k_{\mathrm{a}} c_{\mathrm{M}}(x, t) c_{\mathrm{L}}(x, t)
$$

For the planar case, $\Omega=(0,+\infty)$, the electrode is placed at $x=0$. For spherical case, $\Omega=\mathbb{R}^{3}-S$, the electrode is placed at $S=r \in \mathbb{R}^{3}:|r|<r_{0}$ where $r_{0}$ is the radius of the electrode, usually $5-10 \mu \mathrm{m}$. We shall refer to the electrode surface as $\partial \Omega$ for both planar and spherical geometries. In order to compact the notation, we introduce the functions $c_{\mathrm{X}}=c_{\mathrm{X}}(x, t)$, with $\mathrm{X}=\mathrm{M}, \mathrm{L}, \mathrm{ML}$ to represent the values of the concentrations of the species involved in the processes.

The equilibrium constant between the species of the reaction (11), $K=\frac{k_{\mathrm{a}}}{k_{\mathrm{d}}}$ is defined by:

$$
K=\frac{c_{\mathrm{ML}}^{*}}{c_{\mathrm{M}}^{*} c_{\mathrm{L}}^{*}}
$$

where $c_{\mathrm{x}}^{*}$ are the bulk concentrations of the species.

Initial conditions are the bulk values of $c_{\mathrm{X}}$ :

$$
c_{\mathrm{X}}=c_{\mathrm{X}}^{*} \quad t=0
$$

The boundary conditions for semi-infinite diffusion are the following:

$$
c_{\mathrm{X}} \rightarrow c_{\mathrm{X}}^{*} \quad x \rightarrow \infty
$$

ML and $\mathrm{L}$ are electroinactive, therefore no flux of $\mathrm{ML}$ and $\mathrm{L}$ is crossing the electrode-solution interface $(x=0, \forall t>0)$ :

$$
\left(\frac{\partial c_{\mathrm{ML}}(x, t)}{\partial x}\right)_{x=0}=0 \quad\left(\frac{\partial c_{\mathrm{L}}(x, t)}{\partial x}\right)_{x=0}=0
$$

Finally, the Nernst and flux balance conditions at the electrode surface $\partial \Omega$, in the limit $E-E_{0}<<-0.3 \mathrm{~V}$, give:

$$
c_{\mathrm{M}}(x, t)=0 \quad x=0
$$




\section{The Numerical LBGK Scheme}

The problem stated in equations (6) - (8) is numerically solved by using the Lattice Boltzmann (LB) method in the LBGK approximation [2, 3]. The LB approach is based on a mesoscopic description of the physical system. The space is discretized by a grid or a lattice of spacing $\Delta x$ and a discrete time step of duration $\Delta t$. The lattice is identified by its spatial dimension $d$ and its coordination number $z$ indicating the number of neighbours of each lattice. Traditionally, the lattice is then referred to as a $\mathrm{D} d \mathrm{Q} z$ lattice ( $\mathrm{D}$ stands for Dimension and $\mathrm{Q}$ for Quantities). Important quantities of a LB model are the mesoscopic velocities $v_{i}$, $i=0, \ldots, z$, which denote the admissible velocities of the particles. The admissible velocities reflect the chosen lattice topology so that, in one time step, the particles can reach any of the $z$ neighbouring lattice site. Following the model proposed by [4], the LBGK dynamics of the system is described by the following evolution equation:

$$
f_{i, \mathrm{X}}\left(x+v_{i} \Delta t, t+\Delta t\right)=f_{i, \mathrm{x}}(x, t)+\omega_{\mathrm{X}}\left(f_{i, \mathrm{x}}^{(\mathrm{eq})}(x, t)-f_{i, \mathrm{x}}(x, t)\right)+\Delta t \frac{R(x, t)}{2 d}
$$

where $f_{i, \mathrm{x}}(x, t)$ is the density distribution function, $\omega_{\mathrm{x}}$ is the relaxation parameter, $f_{i, \mathrm{x}}^{(\mathrm{eq})}(x, t)$ is the equilibrium density distribution function and $R(x, t)$ is defined in (9). The second term in the rhs of equation (15) is the non-reactive term, $\Omega_{i, \mathrm{X}}^{\mathrm{NR}}$. The third term in the rhs of equation (15) is the reactive term, $\Omega_{i, \mathrm{X}}^{\mathrm{R}}$. The equilibrium density distribution and the relaxation parameter in equation (15) are 4]:

$$
f_{i, \mathrm{x}}^{(\mathrm{eq})}(x, t)=\frac{c_{\mathrm{X}}}{2 d} \quad \omega_{\mathrm{X}}=\frac{2}{1+\frac{2 d D_{\mathrm{X}} \Delta t}{\Delta x^{2}}}
$$

\subsection{The Time Splitting Method}

In order to perform simulations when the time scale of diffusion and reaction are very different, we will use the time splitting method, following the work presented in [1] wherein the time splitting method has been applied to the LBGK numerical scheme. The complete numerical scheme (15) is now split into its pure diffusive and reactive parts in the following way [1]:

$$
\bar{f}_{i, \mathrm{X}}\left(x+v_{i} \Delta t, t+\Delta t\right)=\tilde{f}_{i, \mathrm{X}}(x, t+\Delta t)+\Omega_{i, \mathrm{X}}^{\mathrm{NR}}(\tilde{f}(x, t+\Delta t))
$$

and

$$
\tilde{f}_{i, \mathrm{X}}\left(x, t^{\prime}+\Delta t_{\mathrm{K}}\right)=\tilde{f}_{i, \mathrm{X}}\left(x, t^{\prime}\right)+\Omega_{i, \mathrm{X}}^{\mathrm{R}}\left(\tilde{f}\left(x, t^{\prime}\right)\right)
$$

Here we have assumed that the time variable $t^{\prime}$ for the reaction equation runs faster than $t$, the time variable of the diffusion equation. This means that $\Delta t_{\mathrm{K}}<<\Delta t$. In addition, the initial condition of equation (18) is $\tilde{f}\left(x, t^{\prime}\right)=$ $\bar{f}(x, t)$. For a detailed description of the technique and of the symbols introduced, see [1]. However, the time splitting method alone is not sufficient to completely solve processes with very large reaction rates. In the next subsection we will explain with a simple example, that applying the time splitting, also requires to adjust the grid size according to the reaction rate. 


\subsection{The Reason to Refine the Grid}

Let us consider a simple reaction diffusion problem with only one species, say $\mathrm{M}$, and let us vary the rate $k$. For a one dimensional problem, considering the initial and the boundary conditions (11) and (12) and the perfect sink condition on $\partial \Omega$, the time splitting exact solution reads [5] in the time interval $[0, t]$ :

$$
c_{\mathrm{M}}(x, t)=c_{\mathrm{M}}^{*} e^{k t} \operatorname{erf}\left(\frac{x}{2 \sqrt{D_{\mathrm{M}} t}}\right)
$$

If we consider the exact solution in two points, $x$ and $x+\Delta x$, we can write:

$$
c_{\mathrm{M}}(x+\Delta x, t)-c_{\mathrm{M}}(x, t)=c_{\mathrm{M}}^{*} e^{k t}\left[\operatorname{erf}\left(\frac{x+\Delta x}{2 \sqrt{D_{\mathrm{M}} t}}\right)-\operatorname{erf}\left(\frac{x}{2 \sqrt{D_{\mathrm{M}} t}}\right)\right]
$$

The first order Taylor series expansion around $x$ of an amount $\Delta x$, with $\Delta x \rightarrow 0$, allows us to write:

$$
c_{\mathrm{M}}(x+\Delta x, t)-c_{\mathrm{M}}(x, t)=c_{\mathrm{M}}^{*} e^{k t} \Delta x \frac{e^{-\frac{x^{2}}{2 \sqrt{D_{\mathrm{M}}} t}}}{\sqrt{\pi D_{\mathrm{M}} t}}
$$

In general, we expect that $c_{\mathrm{M}}(x, t)$ will vary smoothly in space to ensure numerical accuracy. So, if we require that $\left|c_{\mathrm{M}}(x+\Delta x, t)-c_{\mathrm{M}}(x, t)\right|<\theta$, then we obtain the condition:

$$
\Delta x<e^{-k t} \frac{\sqrt{\pi D_{\mathrm{M}} t}}{c_{\mathrm{M}}^{*}} \exp \left(\frac{x^{2}}{2 \sqrt{D_{\mathrm{M}} t}}\right) \cdot \theta
$$

Inequality (22) says that: a) $\Delta x$ has to be bounded and b) if we consider two rates $k_{1}$ and $k_{2}$ such that $k_{1}>k_{2}$, then $\Delta x_{k_{1}}<\Delta x_{k_{2}}$. Therefore, for very fast reaction dynamics, in order to get a variation between $x$ and $x+\Delta x$ less than $\theta$, the grid size $\Delta x$ has to be chosen small enough, accordingly to inequality (22). Furthermore, it is not necessary to use the restriction (22) in all the domain. It is advisable to restrict its usage only to region where this is really needed. In fact, the initial conditions on the concentration profiles $c_{\mathrm{X}}$ are chosen such that equation (10) is fulfilled. This fact suggests to use a grid size that satisfy condition (22) only close to the electrode surface and more precisely within the region comparable to the reaction layer thickness $\mu(\sim 2-3 \mu)$.

\subsection{The Time Splitting - Grid Refinement - LBGK Scheme}

To simplify the description, we will refer only to the simpler refinement made by just two sub-grids. Let us divide the domain $\Omega$ into two sub-domains $\Omega_{1}$ and $\Omega_{2}$. Let us build two grids, $G_{1}$, with discretization parameters $\Delta x_{1}$ and $\Delta t_{1}$, and $G_{2}$, with discretization parameters $\Delta x_{2}=g \Delta x_{1}$ and $\Delta t_{2}=g \Delta t_{1}$, where $g$ is the grid refinement factor. The grid $G_{1}$ covers the set $\Omega_{1}$, while the grid $G_{2}$ covers the set $\Omega_{2}$. The strategy suggested is based on the conservation of the function $c_{\mathrm{x}}$ and of its first derivative $\frac{\partial c_{\mathrm{X}}}{\partial x}$ at the subgrids $\Gamma=G_{1} \cap G_{2}$. If we define $c_{\mathrm{x}}^{(1)}$ 
and $c_{\mathrm{x}}^{(2)}$ as the concentrations defined in the subgrids $G_{1}$ and $G_{2}$ respectively, the conservation conditions are:

$$
c_{\mathrm{X}}^{(1)}=c_{\mathrm{X}}^{(2)} \quad \frac{\partial c_{\mathrm{X}}^{(1)}}{\partial x}=\frac{\partial c_{\mathrm{X}}^{(2)}}{\partial x} \quad x \in \Gamma
$$

which ensures the continuity of the concentration profiles and of the fluxes of the species X. Following [1], the coupling conditions at $x \in \Gamma$ are obtained by solving the following system in the unknowns $f_{1, \mathrm{x}}^{(2)}$ and $f_{2, \mathrm{x}}^{(1)}$ :

$$
\left(\begin{array}{cc}
1 & -1 \\
-\Delta x_{1}-\Delta x_{2}
\end{array}\right)\left(\begin{array}{c}
f_{1, \mathrm{x}}^{(2)} \\
f_{2, \mathrm{x}}^{(1)}
\end{array}\right)=\left(\begin{array}{c}
f_{1, \mathrm{x}}^{(1)}-f_{2, \mathrm{x}}^{(2)} \\
\Delta x_{2}\left[f_{1, \mathrm{x}}^{(1)}-c_{\mathrm{x}}^{(1)}\left(x_{1}\right)\right]+\Delta x_{1}\left[f_{2, \mathrm{x}}^{(2)}-c_{\mathrm{X}}^{(2)}\left(x_{2}\right)\right]
\end{array}\right)
$$

where $x_{1}=x-\Delta x_{1}$ and $x_{2}=x+\Delta x_{2}$. The final numerical scheme in the time interval $\left[t, t+\Delta t_{2}\right]$ is the following:

1. Solve the pure diffusive problem by applying equation (17) in the coarser sub-grid $G_{2}$ and then $g$-times in the finer sub-grid $G_{1}$. Apply the coupling conditions (23) on $\Gamma$ by solving the system (24)

2. Solve the pure reactive problem by applying equation (18) in the coarser sub-grid $G_{2}$ and then in the finer sub-grid $G_{1}$.

\subsection{The Off-Lattice Numerical Boundary Conditions}

In order to preserve the accuracy of the numerical scheme also for irregular boundaries, we propose a numerical discretization of the boundary conditions where the boundary is not on a grid point. For simplicity only discuss the 1D problem. Let us consider the grid $G_{1}$ and two points, $x_{1}$ and $x_{2}$, where $x_{1}$ is inside the boundary, $x_{2}$ outside, and $x_{1}<x_{b}<x_{2}$. The $f_{i, \mathrm{x}}(x, t)$ are computed at $x_{1}$ only to ensure the appropriate boundary condition at $x_{b}$, assuming that interpolation is considered. The Taylor expansion of $c_{\mathrm{X}}$ at $x_{b}$ reads:

$$
c_{\mathrm{X}}\left(x_{2}\right)=c_{\mathrm{X}}\left(x_{b}\right)+\left(\Delta x-x_{b}\right){\frac{\partial c_{\mathrm{X}}}{\partial x}\left(x_{\left(x=x_{b}\right)}\right.}+o\left(\Delta x-x_{b}\right)^{2}
$$

Furthermore, we can write the spatial derivative in the rhs of equation (25) as:

$$
{\frac{\partial c_{\mathrm{X}}}{\partial x}}_{\left(x=x_{b}\right)}=\frac{c_{\mathrm{X}}\left(x_{2}\right)-c_{\mathrm{X}}\left(x_{1}\right)}{\Delta x}+o\left(\Delta x^{2}\right)
$$

By combining expressions (25) and (26) we obtain the numerical Dirichlet and Neumann boundary conditions:

$$
\begin{gathered}
c_{\mathrm{X}}\left(x_{1}\right)=c_{\mathrm{X}}\left(x_{2}\right)-\frac{\Delta x}{\Delta x-x_{b}}\left(c_{\mathrm{X}}\left(x_{2}\right)-c_{\mathrm{X}}\left(x_{b}\right)\right) \\
c_{\mathrm{X}}\left(x_{1}\right)=c_{\mathrm{X}}\left(x_{2}\right)-\Delta x{\frac{\partial c_{\mathrm{X}}}{\partial x}}_{\left(x=x_{b}\right)}
\end{gathered}
$$

All the values of the rhs in equations (27) and (28) are known from the boundary condition or already computed from the numerical scheme. Note that imposing $c_{\mathrm{X}}$ in the LB scheme can be done as explained in [1]. 


\section{Numerical Validation}

We show some numerical validations of the method proposed, for a planar electrode case wherein $D_{\mathrm{M}}=D_{\mathrm{L}}=D_{\mathrm{ML}}=10^{-9} \mathrm{~m}^{2} \mathrm{~s}^{-1}, k_{\mathrm{a}} c_{\mathrm{L}}^{*}=10^{5} \mathrm{~s}^{-1}, k_{\mathrm{d}}=10^{3} \mathrm{~s}^{-1}$. Initial conditions are $c_{\mathrm{M}}^{*}=9.9 \cdot 10^{-6} \mathrm{~mol} \mathrm{~m}^{-3}, c_{\mathrm{ML}}^{*}=9.9 \cdot 10^{-4} \mathrm{~mol} \mathrm{~m}^{-3}$ and $c_{\mathrm{L}}^{*}=10^{-2} \mathrm{~mol} \mathrm{~m}^{-3}$. For this case, the scales are: $\mu=10^{-7} \mathrm{~m}, \delta=5.6 \cdot 10^{-5} \sqrt{t}$ $\mathrm{m}, \frac{1}{k_{\mathrm{a}} c_{\mathrm{L}}^{*}}=10^{-5} \mathrm{~s}, \frac{\delta^{2}}{D_{\mathrm{M}}}=3.14 \cdot t \mathrm{~s}$. The simulations are run up to $t=10^{-2} \mathrm{~s}$. We use the time splitting method by applying the discrete equations (17) and (18) and the reaction is solved with an implicit scheme. We use the grid $G_{1}$ from the electrode surface $(x=0)$ to the distance $x_{b}=3 \mu$ and the the grid $G_{2}$ for $x>x_{b}$. The domain size is $\sim 5 \cdot \delta$ in order to fulfil the semi-infinite diffusion conditions (12).

Tables 1 and 2 show the error on the flux computation computed for several values of the discretization parameters. The relative error $\epsilon$ with respect to an analytical result is computed as in [1].

Table 1 shows that the error decreases if the grid size close to the electrode surface becomes smaller, until $\Delta x_{1}=\mu / 20$. For smaller grid sizes, the error increases again, due to the small values of the relaxation parameter. In fact, when $\Delta x_{1}=\mu / 50$, the corresponding values of $\omega_{1}$ and $\omega_{2}$ are too small and the pure diffusive scheme loses accuracy. It is reasonable to fix the relaxation parameters in the range $0.8<\omega<1.5$ to get the best performance. A way to circumvent this problem would be to have a grid refinement technique where $\frac{\Delta x^{2}}{\Delta t}$ is constant across the grid instead of $\frac{\Delta x}{\Delta t}$. This topic is under investigation.

Table 2 shows the effect of different grid refinement factors, $g$, for $\Delta x_{1}=\mu / 20$ and $\Delta x_{2}=g \Delta x_{1}$, on accuracy and CPU time. The case where only $G_{1}$ and $G_{2}$ are used in all the domain, is shown for comparison. If $g$ increases, then the error increases as well, but the computation time is shorter. However, for $g=8$ the error is more than 5 percent, a value no longer acceptable. It is preferable to use $g=2-4$ which allows us to gain time of computation without losing too much in precision. It is interesting to compare the errors and the computation time shown for several $g$ with the results obtained by applying only the grid $G_{1}$ everywhere. The results show that the grid refinement allows us to reduce the computation time while keeping a good precision. With $g=2$, the error still remains less than $1 \%$ and the computation time is reduced $20 \%$ in comparison to the time required using only the grid $G_{1}$. Furthermore, in the last line of table 2 ,

Table 1. Error for different space grid sizes. Parameters of the simulation: $D_{\mathrm{M}}=$ $D_{\mathrm{L}}=D_{\mathrm{ML}}=10^{-9} \mathrm{~m}^{2} \mathrm{~s}^{-1}, k_{\mathrm{a}} c_{\mathrm{L}}^{*}=10^{5} \mathrm{~s}^{-1}, k_{\mathrm{d}}=10^{3} \mathrm{~s}^{-1} \cdot g=2, \Delta x_{2}=g \Delta x_{1}, x_{b}=3 \mu$, $t=10^{-2}$ s. (1000 time steps).

\begin{tabular}{cccc}
\hline$\Delta x_{1}$ & $\omega_{1}$ & $\omega_{2}$ & $\epsilon$ \\
\hline$\mu / 5$ & 1.90 & 1.95 & $8.71 \%$ \\
$\mu / 10$ & 1.67 & 1.82 & $3.80 \%$ \\
$\mu / 20$ & 1.11 & 1.43 & $0.50 \%$ \\
$\mu / 50$ & 0.33 & 0.57 & $3.90 \%$ \\
\hline
\end{tabular}


Table 2. Error for different grid refinement factors $g$. Same parameters as in table 1 but $\Delta x_{1}=\mu / 20$ and $\Delta x_{2}=g \Delta x_{1}$.

\begin{tabular}{ccc}
\hline$g$ & Computation time & $\epsilon$ \\
\hline 2 & 0.80 & $0.50 \%$ \\
4 & 0.77 & $2.03 \%$ \\
6 & 0.72 & $4.79 \%$ \\
8 & 0.70 & $7.52 \%$ \\
\hline only grid $G_{1}$ everywhere & 1 & $0.02 \%$ \\
\hline only grid $G_{2}(g=2)$ everywhere & 0.50 & $0.71 \%$ \\
\hline
\end{tabular}

we show the error and the computation time obtained with $\Delta x_{2}=2 \Delta x_{1}$, by applying only the grid $G_{2}$. The computation time is still reduced, but the error obtained by using only the coarser grid everywhere is larger.

\section{Conclusions}

We have seen that for processes involving fast reactions the grid size should be small enough to follow the strong variations of the concentration profiles close to the electrode surface. However, the grid size should be chosen carefully in order to avoid values of the relaxation parameters too small. It is advisable to choose grid size values, such that the corresponding relaxation parameter values range between 0.8 and 1.5. The grid refinement is very useful because it allows us to keep a good precision and to reduce the time of computation.

\section{Acknowledgements}

The authors gratefully acknowledge support of this work by the Swiss National Foundation. Josep Galceran also thanks the support of this research by the Spanish Ministry of Education and Science (DGICYT: Project BQU2003-07587).

\section{References}

1. Alemani, D., Chopard, B., Galceran, J., Buffle, J.: Phys. Chem. Chem. Phys., 7 (2005) 3331-3341.

2. Wolf-Gladrow, D.A.: Lattice Gas Cellular Automata and Lattice Boltzmann Models: An introduction, Printed in Berlin, Springer-Verlag (2000).

3. Chopard, B., Droz, M.: Cellular Automata Modelling of Physical Systems, Cambridge Univ. Press. UK (1988).

4. Dawson, S., Chen, S., Doolen, G.D.: Journal of Chemical Physics 98 (1992) 1514.

5. Hundsdorfer, W., Verwer, J.G.: Numerical Solution of Time-Dependent AdvectionDiffusion-Reaction Equations, Printed in Berlin, Springer-Verlag (2003). 